\title{
EL NEGOCIO INCLUSIVO DE RECICLAJE ENTENDIDO COMO UNA ESTRATEGIA EMPRESARIAL A DESARROLLAR POR LA INDUSTRIA MANUFACTURERA DE PAPEL Y CARTÓN EN LA CIUDAD DE CUENCA - ECUADOR.
}

THE INCLUSIVE RECYCLING BUSINESS UNDERSTOOD AS A BUSINESS
STRATEGY TO BE DEVELOPED BY THE MANUFACTURING INDUSTRY
PAPER AND CARDBOARD IN THE CITY OF CUENCA - ECUADOR.

José Santiago Jimbo Días ${ }^{1}$

santiago.jimbo@ucuenca.edu.ec

\section{Forma sugerida de citar:}

Jimbo Días, Santiago (2016). El negocio inclusivo de reciclaje entendido como una estrategia empresarial a desarrollar por la Industria Manufacturera de papel y cartón en la ciudad de Cuenca - Ecuador. Revista Economía y Política, Año XII, No. 24. pp. 147-167

Fecha de recepción: 19 de noviembre de 2016

Fecha de aceptación: 30 de marzo de 2017

\begin{abstract}
This article deals with the evolution of the Economic Pyramid (BDP) in Latin America, the Caribbean and its incidence in Ecuador, which contributes to the inclusion of cluttering business $(\mathrm{NI})$ that collect paper and cardboard which is being recycled whose contribution is taken advantage by the manufacturing Industry in the Austro Region of Ecuador (Cuenca), making it possible to consider an $\mathrm{NI}$ as a contribution to the global trend in inclusion of the poor according to their annual income per project based on the parity of their purchasing power (PPA), considering its members as strategic partners to fulfill the activities that contribute to the industry's value chain, while providing the possibility of having an income to cover some of their basic needs.
\end{abstract}

KEYWORDS: Economic Pyramid Base, Inclusive Business, Business Strategy, Creating Shared Value.

PALABRAS CLAVES: Base de Pirámide Económica, Negocio Inclusivo, Estrategia Empresarial, Creación de Valor Compartido.

JEL: L67, M11

1 Ingeniero Industrial por la Universidad de Cuenca. Máster en Administración de Empresas. Docente Titular e Investigador por la Facultad de Ciencias Económicas y Administrativas. Líneas de Investigación en Gestión Empresarial, Estrategia Competitiva, Negocios Inclusivos y Medio Ambiente. 


\section{Introducción}

El presente artículo tiene como punto de partida la Pirámide Económica que fue propuesta por (Prahalad \& L.Hart, 2002) en cuya base se encuentran 4.000 millones de personas con ingresos per cápita anuales inferiores a $\$ 1500$ dólares, que no son atendidas por las empresas en lo que se refiere a oferta de bienes o servicios para satisfacer sus necesidades, justamente por su situación económica, lo que ha provocado que exista una priorización al concentrarse en quienes más ingresos poseen, considerándolos como los únicos que pueden pagar, volcando todos los esfuerzos para satisfacer sus necesidades.

Sin embargo, en la actualidad la base de la pirámide (BDP) ha evolucionado adquiriendo especificidades a tal punto que para América Latina, Caribe y Ecuador su composición es diferente a otras partes del planeta, por lo tanto fue necesario puntualizar particularidades en donde se consideró a la BDP como el origen de los Negocios Inclusivos (NI), que se pueden traducir en las estrategias bajo las cuales se incluye a la población de la BDP como socios, proveedores, consumidores de bienes o servicios que puede ofrecer una empresa.

Con la descripción que se realizó de la evolución de la BDP y su relación directa con los $\mathrm{NI}$, se evidencio la oportunidad de realizar una profundización a futuro mediante una investigación científica sobre este NI en el Ecuador aplicada en la ciudad de Cuenca, por intermedio de la industria manufacturera de papel y cartón al incorporar a la población de la BDP mediante el $\mathrm{NI}$ de reciclaje de papel y cartón al considerar a sus integrantes como socios estratégicos, al ser proveedores de materia prima ( papel y cartón reciclados ) bajo la figura de recicladores, permitiéndoles a su vez disponer de ingresos que les brinde la posibilidad de poder adquirir bienes o servicios que necesitan.

Esta interacción de la población de la BDP a partir del NI de reciclaje con la industria manufacturera de papel y cartón se indicó que se puede lograr gracias a la Renarec ( Red Nacional de Recicladores del Ecuador) la cual permite a sus miembros mayor posibilidad de la consecución de sus objetivos al estar fortalecidos como organización y contar con una estructura que facilite su crecimiento y poder de negociación, no obstante se describió que su desarrollo también depende de las políticas del Estado Ecuatoriano, así como también de las ordenanzas del Gobierno Autónomo Descentralizado Municipal de Cuenca, quienes al establecer dentro de sus estrategias o planes de trabajo la prioridad a la gestión integral de los residuos sólidos, generan el contexto para el crecimiento tanto del NI de reciclaje así como de la Renarec. 
La descripción final que se realizo fue pensar en el $\mathrm{NI}$ de reciclaje como una estrategia empresarial, pero vista desde la perspectiva de creación de valor compartido, que implica unir el mundo de los negocios con la sociedad, creando valor económico al mismo tiempo de crear valor para la sociedad (Muñoz, 2013), de esta manera se incluye como un actor determinante a la empresa, indicando la necesidad de un cambio de paradigma en el cual se deje de pensar solamente en los ingresos y que a la par se considere el bienestar de la sociedad, sin que esto perjudique la actividad empresarial.

\section{La base de la pirámide económica, el origen del concepto de los Negocios Inclusivos}

El concepto de la base de la pirámide económica (BDP) fue desarrollado por (Prahalad \& L.Hart, 2002), quienes indican según datos de U.N. World Development Reports que la población mundial se puede dividir en niveles de acuerdo a su ingreso anual per cápita basado en la paridad de poder adquisitivo (PPA), para lo cual se utiliza el gráfico de una pirámide para representar los rangos de ingresos, estando el primer nivel constituido por 75 a 100 millones de personas con ingresos de más de $\$ 20.000$ al año, en el medio de la pirámide se encuentran los niveles 2 -3 que están constituidos por 1500 a 1750 millones de personas con ingresos en un promedio de \$1500 a \$20000 dólares al año y en el nivel 4 al cual se lo denomino la base de la pirámide (BDP) se encuentran 4 mil millones de personas cuyo ingreso es inferior a $\$ 1500$ dólares al año.

\section{Imagen 1:}

\section{La base de la Pirámide Económica}

\begin{tabular}{|c|c|c|}
\hline Ingreses Anuales Per Capita & Niveles & Potiscibn en millenes \\
\hline Más de $\$ 20,000$ & 1 & $75-100$ \\
\hline$\$ 1,500-\$ 20,000$ & 283 & $1.500-1.750$ \\
\hline Menos de $\$ 1.500$ & 4 & 4.000 \\
\hline
\end{tabular}

FUENTE: (Prahalad \& L.Hart, 2002)

Esta representación gráfica permitió visibilizar en la BDP, un mercado desatendido por parte de las empresas debido a su condición económica, la cual impedía el adquirir bienes o servicios ofertados, sin embargo, la cantidad de población que la integra según (Prahalad 
\& L.Hart, 2002) debe ser de interés de las empresas al considerarla como un mercado potencial para ganar dinero ofreciéndoles bienes y servicios a los que actualmente no pueden acceder.

Al haber desarrollado el concepto de la BDP y la importancia de incluir a la población que está dentro de ella como un mercado a atender por parte de las empresas, hizo necesario pensar en las formas de inclusión y que las mismas sean beneficiosas tanto para las empresas como para esta población, de esta manera (Prahalad \& Hammond, 2005), la visualizan no como individuos, sino como una comunidad, donde su poder de compra al operar de esta manera es representativa, sumado a este factor que son mercados que se encuentran en las primeras fases del desarrollo económico, lo cual provocaría un crecimiento de las empresas que apuesten a ingresar en este mercado de una manera acelerada ya no solo considerando a esta población como consumidores, sino también como colaboradores o socios estratégicos que contribuyan a reducir costos al transferir eficiencias e innovaciones en el modelo de negocio, no obstante para lograr dichos propósitos es necesario un cambio en el paradigma mental de los inversionistas y empresarios para que visibilicen las potencialidades empresariales que pueden ofrecer las personas con bajos ingresos.

Cada uno de los planteamientos antes citados contribuyeron para la generación del concepto de negocios inclusivos (NI) al cual se lo puede definir como: Estrategias empresariales basadas en emprendimientos, económicamente rentables, social y ambientalmente responsables, para atender los problemas de pobreza, con la participación activa de las empresas en su disminución y en la generación modelos de negocios comprometidos con la responsabilidad social y la inclusión de las personas pobres, donde desarrollen sus capacidades ya no solo viéndolos como consumidores, sino como colaboradores en la cadena de valor de las empresas. (Prahalad \& Hammond, 2005) (AVINA, 2010) (Licandro, El rol de las alianzas intersectoriales en la creacion de negocios inclusivos con la base de la pirámide, un análisis de la experiencia uruguaya mediante el estudio de casos, 2013) (Pineda Escobar \& Falla Villa, 2016).

Este concepto y de la BDP se han ido reforzando y la vez evolucionando a nivel mundial con el paso del tiempo, América latina y el Caribe no han sido la excepción, más aun al ver en ellos la oportunidad para combatir la pobreza, esto hace necesario el ir adentrándose en los $\mathrm{NI}$ y las personas que se encuentran en la BDP en América latina y el Caribe para conocer su descripción, análisis y características propias con el propósito de medir el impacto de su aplicación, de esta manera según el (Banco Interamericano de Desarrollo, 2015) se evidencia una actualización en la orientación que se debe dar a los NI y a las personas que se encuentran en la BDP pasando de entenderlos como un grupo homogéneo 
de personas de bajos ingresos sin el poder adquisitivo suficiente para ser un mercado de inversiones de negocio sostenibles, a un grupo de personas con mayores oportunidades de desarrollo que se evidencian en la urbanización de las ciudades, el incremento en los ingresos y el acceso a educación y tecnología.

De esta manera el mercado para los NI en función a la BDP en América Latina y el Caribe está compuesto por hogares cuyos miembros cuentan con ingresos de hasta US\$10 diarios per cápita, medido en paridad de poder adquisitivo (PPA) en el 2005 y representan alrededor del $70 \%$ de la población de la región, que equivale a 405 millones de personas. A diferencia de la BDP de otras regiones del mundo, la BDP de América Latina y el Caribe es urbana en su mayoría: tres cuartas partes de los hogares de la BDP de América Latina y el Caribe están ubicados en zonas urbanas y tienen acceso a servicios básicos, incluidos agua y electricidad. (Banco Interamericano de Desarrollo, 2015)

Este mercado está compuesto por dos segmentos clasificados de acuerdo a sus ingresos per cápita basado en la paridad de poder adquisitivo (PPA) recibiendo la denominación de: pobre, que vive con hasta US\$4 diarios per cápita en PPA y que equivale a 183 millones de personas, mientras tanto el vulnerable, que vive con un rango de US $\$ 4$ a US $\$ 10$ diarios per cápita en PPA que equivale a 222 millones de personas. Recibiendo la denominación de hogares pobres aquellos que no cuentan con los recursos ni capacidades suficientes para satisfacer sus necesidades actuales, mientras que los hogares vulnerables son aquellos que, si bien pueden satisfacer algunas necesidades, aún están en riesgo de caer en la pobreza. (Banco Interamericano de Desarrollo, 2015).

El conocer la población que integra la BDP de América Latina y el Caribe permite identificar el mercado potencial que puede ser explotado por las empresas y la aplicación de los NI el mismo que representa 759 mil millones de dólares anuales, de los cuales 174 mil millones de dólares son proporcionados por la población pobre (183 millones de personas) y 585 mil millones de dólares los generan la población vulnerable (222 millones de personas), estos valores se los obtiene de los patrones de consumo de la población de la BDP cuyos egresos no se limitan a los bienes de consumo o necesidades básicas, debido a que el $27,5 \%$ de los gastos se lo emplea en comida y el resto se lo utiliza en vivienda, transporte, entretenimiento, educación, etc.

Cabe recalcar que entre los años 2000 y 2010 hubo un incremento en todas las categorías de gasto provocado por el mejoramiento en los ingresos de la población de la BDP, sin embargo en el año 2010 dentro estas categorías se puedo evidenciar una disminución en gastos de productos de primera necesidad, servicios y un incremento en bienes y servicios discrecionales, en el siguiente gráfico se indican los gastos y por ende los potenciales 
mercados a explotar de la población de la BDP en América Latina y el Caribe para el año 2010. (Banco Interamericano de Desarrollo, 2015)

\section{Gráfico 2.}

Mercados potenciales de la población de la BDP en América Latina y el Caribe para el año 2010.

\begin{tabular}{|l|r|}
\hline \multicolumn{2}{|c|}{ Mercados de la BDP } \\
\hline \multicolumn{1}{|c|}{ Mercados Potenciales } & $\begin{array}{c}\text { Mil Millones } \\
\text { de dólares }\end{array}$ \\
\hline Comida & $\$ 209$ \\
Vivienda & $\$ 184$ \\
Transporte & $\$ 82$ \\
Servicios Alimentación & $\$ 44$ \\
Vestimenta & $\$ 42$ \\
Otros & $\$ 40$ \\
Energía & $\$ 38$ \\
Salud & $\$ 31$ \\
Educación & $\$ 29$ \\
TIC & $\$ 25$ \\
Recreación & $\$ 14$ \\
Servicios Financieros & $\$ 11$ \\
Agua & $\$ 10$ \\
\hline TOTAL & $\$ 759$ \\
\hline
\end{tabular}

FUENTE: (Banco Interamericano de Desarrollo, 2015) ELABORACIÓN: Propia

Los datos antes indicados reflejan tanto una población en millones de personas, así como también un mercado detallado de categorías de posibles oportunidades para los $\mathrm{NI}$ medidos en millones de dólares en América Latina y el Caribe, brindando la pauta de la aplicación de los conceptos de la BDP y de los NI en el Ecuador, país en el cual se puede evidenciar que dentro del 2000 al 2010 la población BDP aumentó 3\% de 11,2 millones a 11,6 millones de personas, estando constituida por población pobre (aquellos con ingresos de menos de US\$4 por día) que cayó 34\% de 8 millones a 5,3 millones y la población vulnerable (aquellos con ingresos entre US $\$ 4$ y US $\$ 10$ por día) la cual aumentó $97 \%$ es 
decir de 3,2 millones a 6,3 millones, manteniendo la tendencia antes descrita en América Latina y el Caribe. (Banco Interamericano de Desarrollo, 2015)

Esta información se ve reforzada a nivel del Ecuador por datos del (INEC, 2015) en la sección de proyecciones por edades provincias 2010-2020 y nacional, donde se indica que para el año 2020 la población total del Ecuador será de 17.510 .643 habitantes lo cual representa un crecimiento muy importante si se compara la cantidad actual de 16.528 .730 habitantes, siendo más específicos para este análisis y para el posterior planteamiento de la aplicación del concepto de la BDP y los NI, la población a ser analizada es la ciudad de Cuenca, que pertenece a la provincia del Azuay o Región Austro del país, que para el año 2020 tendrá un crecimiento de 112.433 habitantes de la cantidad actual que es 524.563 habitantes, mientras que la provincia del Azuay pasara a tener 881.394 habitantes con un incremento de 70.982 habitantes.

Esto implica un reto para la región del Austro en especial para la ciudad de Cuenca de conseguir un crecimiento poblacional que vaya a la par con la disminución de la pobreza, bajo las perspectivas de la BDP y los $\mathrm{NI}$ que según el reporte de pobreza por ingresos a junio del 2015 elaborado por el INEC, utilizando la misma denominación del Banco Interamericano de Desarrollo los ingresos de la población vulnerable a junio del 2015 se ubicó en $\$ 83,29$ mensuales por persona y en la población pobre sus ingresos eran de $\$ 46,94$ mensuales por persona, en lo que respecta al país. Para la ciudad de Cuenca se registra un nivel de población vulnerable de 5,57\%, y en lo que hace referencia a población pobre existe un leve aumento que corresponde a 0,77\% para Junio del 2015 en comparación del 0,58\% de Junio del 2014, aunque estas son variaciones no significativas estadísticamente.

Estos valores hacen necesario que el Ecuador y la ciudad de Cuenca empiecen aplicar el concepto de los NI en la BDP, como una estrategia para afrontar la pobreza de acuerdo a los ingresos, debido a que según los datos antes indicados la población pobre descendió pasando a formar parte de la población vulnerable, por lo tanto si no se toman las medidas correctas esta población podría regresar nuevamente a formar parte de la pobre con el atenuante que se habría incrementado, sin embargo los conceptos de la BDP y de NI no solo son responsabilidad de las empresas también incluyen a los gobiernos de cada país, de esta manera en el Ecuador su aplicación está sustentada por el Plan Nacional del Buen Vivir 2013-2017 al alinearse de forma directa con el objetivo 9 al Garantizar el Trabajo Digno en todas sus Formas, donde se reconoce la supremacía del trabajo humano sobre el capital de forma incuestionable y por lo tanto se considera que el trabajo no puede ser concebido como un factor más de la producción sino como elemento mismo del Buen Vivir en el Ecuador, generando de esta manera el contexto necesario para su aplicación. 


\section{Modalidades de los negocios inclusivos y su aplicación en la ciudad de Cuenca.}

Como un paso previo del análisis de la aplicación de los NI en la ciudad de Cuenca - Ecuador, es necesario conocer algunas experiencias a nivel mundial que han tenido gran impacto y aceptación tanto en personas de la BDP así como en las empresas, este es el caso de Nicaragua y Honduras por intermedio de la empresa Mercon Coffee Group, MCG, al considerar a las personas de bajos ingresos como socios empresariales, proveedores de materia prima, reduciendo los costos de producción y generando empleos. Mercon Coffee Group, MCG, está constituida por empresas de gran reconocimiento en el mundo que se dedican al negocio de la comercialización y exportación de café en los mercados de los Estados Unidos de Norteamérica y del Reino Unido principalmente, estando ubicadas sus empresas en Nicaragua, Honduras, Guatemala, México, Vietnam, Nueva York y Londres. (Netherlands Development Organisation (SNV); World Business Council for Sustainable Development, 2010)

Bajo el concepto de los $\mathrm{NI}$ de ser estrategias empresariales para atender los problemas de pobreza, con la participación activa de las empresas en su disminución y en la generación de modelos de negocios comprometidos con la responsabilidad social, ambiental y la inclusión de las personas pobres, sus empresas Comercial Internacional Exportadora S.A., CISAExportadora, y Comercial Internacional de Granos de Honduras, CIGRAH, han desarrollado $\mathrm{NI}$ en las principales zonas cafeteras de Nicaragua y Honduras respectivamente, mediante la vinculación de proveedores o socios estratégicos a pequeña escala, a quienes prestan asistencia técnica integral en aspectos de buenas prácticas de manufactura, estándares de calidad, procesos de trazabilidad e incorporación de certificaciones sostenibles, permitiendo a la empresa acceder a los mercados que brindan un tratamiento preferencial al café que cumple con esta clase de atributos. (Netherlands Development Organisation (SNV); World Business Council for Sustainable Development, 2010)

Este NI permite responder al incremento de la demanda mundial de café certificado, las empresas de MCG buscan aumentar su oferta en el mercado internacional, diferenciada con el distintivo Utz Certified, sello internacional que se basa en un código de conducta para la producción social y ambientalmente sostenible, lo cual permite la obtención de mejores precios de venta, la aplicación del sello ha significado un incremento en el abastecimiento de café certificado y por lo tanto un aumento en los ingresos de CISA-Exportadora, de CIGRAH y de los proveedores 0 socios estratégicos de las zonas cafeteras de Nicaragua y Honduras, quienes obtienen una mayor utilidad al aumentar la calidad de su café y la productividad de sus fincas. (Netherlands Development Organisation (SNV); World Business Council for Sustainable Development, 2010) 
Otro caso de aplicación de los NI en la BDP, es el denominado Ave Fénix, en Uruguay que comenzó a trabajar en la Costa de Oro del departamento de Canelones el 15 de enero de 2007, constituyéndose jurídicamente como cooperativa social el día 27 de junio de 2008. Fue el primer grupo asociativo de recicladores del departamento de Canelones y se creó en el marco de la Ley de Uso de Envases No Retornables 117, con un plan integral de gestión de envases que se ha desarrollado bajo la consigna ¡Tu Envase Nos Sirve! (Licandro \& Pardo, Experiencias de Negocios Inclusivos en el Uruguay, 2013)

Participaron en este proceso la Intendencia Municipal de Canelones (IC), el Ministerio de Desarrollo Social (MIDES) a través del Programa Uruguay Clasifica (PUC), el Ministerio de Vivienda Ordenamiento Territorial y Medio Ambiente (MVOTMA) a través de la Dirección Nacional de Medio Ambiente (DINAMA), la Cámara de Industrias del Uruguay, la ONG, Centro Uruguay Independiente (CUI) y la cooperativa de recicladores Ave Fénix. (Licandro \& Pardo, Experiencias de Negocios Inclusivos en el Uruguay, 2013)

Su constitución permitió Ave Fénix realizar la recolección de residuos con un camión facilitado por la IC, siendo los materiales recuperados de islas de reciclaje colocadas en supermercados de la Ciudad de la Costa y Costa de Oro. Este método fue sustituyendo paulatinamente la recolección puerta a puerta y será incorporado gradualmente en el resto del departamento, esto se debe a la comparación realizada con el servicio municipal de recolección puerta a puerta, el cual resultaba ineficiente debido a que la zona de la Costa es una ciudad con gran concurrencia de turistas, lo cual generaba problemas de continuidad de la recuperación de material reciclable, debido a la imposibilidad de establecer horas para brindar el servicio en las que las personas estén en su casa en el momento de la recolección. (Licandro \& Pardo, Experiencias de Negocios Inclusivos en el Uruguay, 2013)

Los materiales recuperados son transportados a los centros de acopio, allí se pesan y se trasladan para realizar su clasificación obteniéndose como valores representativos en volumen, el cartón $34 \%$, el vidrio $31 \%$ y el PET $20 \%$, luego de este proceso, se vuelven a pesar, con el objetivo de calcular el rendimiento y la cantidad de materiales recuperados para finalmente embolsarlos para la venta, la cual se realiza a intermediarios, generalmente cada 15 días. En este NI la figura del intermediario es consecuencia de la pequeña escala y carencia de infraestructura de cada reciclador, inclusive de las propias cooperativas, que les impide acceder directamente, a los compradores finales ( industrias que reciclan) debido a que estas industrias compran a gran escala, requiriéndose por lo tanto infraestructura, logística, vehículos y capital (para financiar la compra de materia prima, almacenaje y fletes), que los recicladores individuales y cooperativas de recicladores no poseen. (Licandro \& Pardo, Experiencias de Negocios Inclusivos en el Uruguay, 2013) 
Con la experiencia ganada en la implementación de este $\mathrm{NI}$ y con el paso de los años ha hecho necesario diversificar las materias primas que clasifica y comercializa, pasando a realizar el reciclaje de todo tipo de materiales (envases y no envases), a la vez de agregarles valor con procesos como picado, prensado, etc. A partir de esta redefinición del negocio, Ave Fénix ha incrementado en cerca de un $80 \%$ sus ingresos, si se comparan las cifras de 2011 con las del mismo mes del año anterior, siendo los meses de verano en donde se recoge más material, constituyendo un apoyo al crecimiento de este $\mathrm{NI}$ el marco jurídico de la Ley de Envases y la política municipal en materia de recolección de residuos, además de la visión de haber aplicado la diversificación los materiales que se reciclan y los servicios agregados en los mismos posibilitando la sostenibilidad económica. (Licandro \& Pardo, Experiencias de Negocios Inclusivos en el Uruguay, 2013)

Los casos antes citados contribuyen a fortalecer la oportunidad y necesidad a nivel de Ecuador y de la ciudad de Cuenca para la aplicación de los NI en función a la BDP, siendo trascendente describir que existen dos modalidades en las que los $\mathrm{NI}$ proponen vincular a las personas de bajos ingresos con las empresas: la primera modalidad es cuando la empresa las incorpora como socios empresariales, proveedores de materia prima, de servicios o distribuidores, reduciendo los costos de producción y generando empleos. La segunda modalidad es como consumidores cuando la empresa pone en el mercado bienes y servicios que satisfacen las necesidades de las personas de bajos ingresos en condiciones que puedan acceder a ellos. (Banco Interamericano de Desarrollo, 2015) (Licandro, El rol de las alianzas intersectoriales en la creacion de negocios inclusivos con la base de la pirámide, un análisis de la experiencia uruguaya mediante el estudio de casos, 2013) (Prahalad \& Hammond, 2005) (Pineda Escobar \& Falla Villa, 2016)

Con la descripción de las modalidades bajo las cuales se pueden crear NI en la BDP del Ecuador es necesario determinar qué tipo de $\mathrm{NI}$ se ha venido generando o que se puede desarrollar en la ciudad de Cuenca, entendiendo que su aplicación es diversa y que como se indicó en párrafos anteriores existe un mercado de 759 millones de dólares en diferentes categorías de bienes o servicios que se podrían analizar, sin embargo y al hacer una fusión de las modalidades de los NI que permiten incluir a la población de la BDP como socios, proveedores, distribuidores y a la vez consumidores su aplicación se la analiza por medio de la perspectiva del reciclaje considerando también para esta elección la premisa del concepto de NI de ser ambientalmente responsable.

Convirtiéndose de esta manera el presente artículo en una introducción a una investigación científica posterior con respecto al NI de reciclaje de papel y cartón en la ciudad de Cuenca - Ecuador, del cual como se abordara en el desarrollo de este artículo, no se dispone de información suficiente a pesar de estar enmarcado en la tendencia mundial de la BDP, esta 
falta de información no hace posible desarrollar su implementación a gran escala y tampoco permite conocer sus beneficios, los cuales serían tanto para la población de la BDP como para la industria manufacturera de papel y cartón, sin embargo, estos actores no son los únicos beneficiados de su aplicación, porque como se revisó en el caso de la cooperativa Ave Fénix ubicada en Uruguay, también los son organismos gubernamentales como el Ministerio del Ambiente del Ecuador (MAE), Gobierno Autónomo Descentralizado Municipal de Cuenca por el intermedio de su Empresa Pública Municipal de Aseo de Cuenca (EMAC) etc.

Otro aspecto a considerar es la dificultad de fortalecer la relevancia social que tiene este $\mathrm{NI}$ en lo que respecta a visibilizar y potencializar la gestión y organización de la Red Nacional de Recicladores del Ecuador (RENAREC) sede Cuenca, de la cual sus particularidades serán explicadas a lo largo de este artículo, la relevancia cognitiva es otro elemento que no se puede desarrollar en referencia a la aplicación de este $\mathrm{NI}$, debido a que no se cuenta con teorías ni estudios sobre esta estrategia empresarial en la ciudad de Cuenca y su relación directa con la BDP.

Esto da paso a la generación de la interrogante de investigación, ¿Cuáles son las fortalezas, debilidades, oportunidades y amenazas del $\mathrm{NI}$ de reciclaje de papel y cartón en la ciudad de Cuenca -Ecuador?, mediante la cual se pretende generar alcance o impacto teórico contribuyendo al entendimiento y comprensión general de los NI de reciclaje. Siendo el objetivo central de la investigación el analizar el impacto que ha tenido en la población de la ciudad de Cuenca la implementación del NI de reciclaje de papel y cartón, mediante características organizacionales de los recicladores y la identificación de su estructura organizacional, las fortalezas, debilidades, oportunidades y amenazas como organización. (Ynoub, 2015)

La metodología que será empleada para realizar una profundización en este $\mathrm{NI}$ es un esquema de investigación Descriptivo-Correlacional, Cuali-Cuanti, siendo las unidades de análisis los recicladores de la ciudad de Cuenca, la industria manufacturera de papel y cartón y la RENAREC, con un diseño multivariado, muestras intensivas con un tratamiento transversal, debido a que las variables de las unidades de análisis podrán ser, por parte de los recicladores la cantidad, el tipo o precio de material reciclado, sus características sociodemográficas, económicas, etc. (Ynoub, 2015)

Mientras que para la industria manufacturera de papel y cartón se considerarían variables como: toneladas de papel que se recuperan por reciclaje, reducción en importaciones de papel reciclado, disminución de costos de producción, etc., denominándose muestras intensivas debido a que las unidades de análisis serían: los recicladores y la industria 
manufacturera de papel y cartón y su transversalidad se debe a que se la realizara para el momento actual, de esta manera en los siguientes párrafos se describe el contexto en el cual se trabajaría la investigación científica. (Ynoub, 2015)

Por lo tanto, se parte señalado que el reciclaje se lo puede denominar como un NI debido a que lo realizan las personas de la BDP en articulación con la empresa privada y con el apoyo de la generación de leyes u ordenanzas emitidas por el Estado Ecuatoriano y Gobiernos Autónomos Descentralizados Municipales (GADM), que permiten a esta población tener recursos económicos para acceder a bienes y servicios, este NI inclusivo empieza a formalizarse a nivel del Estado Ecuatoriano desde la creación del programa nacional para la gestión integral de residuos sólidos (PNGIDS) en el año 2010 desarrollado por el Ministerio del Ambiente para posibilitar la coordinación de actividades y acciones entre el gobierno central, gobiernos locales, empresas públicas y privadas, organizaciones civiles, etc. A esto se suma la generación del programa de la nueva matriz productiva del Ecuador en marzo del 2015 donde se propicia el impulso a la cadena de gestión integral de residuos sólidos (GIRS), potencializando de esta manera al $\mathrm{NI}$ de reciclaje como una alternativa para los procesos productivos y económicos del Ecuador. (Inicitiva regional para el reciclaje inclusivo, 2014-2015)

No obstante, la visibilización de la población involucrada en el $\mathrm{NI}$ de reciclaje se inicia desde el año 2008 con la conformación de RENAREC, fundada el 12 de Diciembre del 2008 en Cuenca, actualmente está integrada por 38 asociaciones de recicladores a nivel nacional, tanto de la Costa como de la Amazonía y en su mayoría de la Sierra, cuenta actualmente con 200 mil recicladores en el país y sus respectivas familias quienes se dedican al trabajo de reciclaje de residuos sólidos en: calles, rellenos sanitarios, botaderos, escombreras y centros de acopio, etc. (Renarec, 2015)

Su creación formal como organización le ha permito la obtención de logros como la firma del convenio en el año 2014 de cooperación conjunta con el gobierno nacional a través de los Ministerios de Ambiente (MAE), de Inclusión Económica y Social, MIES y el Instituto de Economía Popular y Solidaria, IEPS, para aportar al proceso de desarrollo de políticas públicas que permitan el aprovechamiento de residuos sólidos con inclusión y la generación de programas y proyectos específicos en diferentes municipios del país, permitiendo a la RENAREC plantearse propósitos tales como el transformarse en una Federación de Recicladores del Ecuador, cuidar el medio ambiente y contar con una política nacional por la inclusión social y económica de los recicladores. (Renarec, 2015)

Todas estas acciones se las han realizado en un contexto favorable, debido al potencial que existe en el Ecuador en materia de reciclaje, el cual se lo puede evidenciar al hacer el 
análisis de la información del Ministerio de Ambiente por medio del programa nacional para la gestión integral de desechos sólidos (MAE-PNGIDS), donde se indica que en los 221 Gobiernos Autónomos descentralizados Municipales (GADM) se genera un aproximado de 4,1 millones de toneladas métricas al año de residuos sólido urbanos, de los cuales el $61,4 \%$ son orgánicos, papel y cartón $9,4 \%$, plástico $11 \%$, vidrio $2,6 \%$, chatarra $2,2 \%$, y otros 13,3\%. (Inicitiva regional para el reciclaje inclusivo, 2014-2015). Estos datos reflejan la oportunidad para el desarrollo del $\mathrm{NI}$ de reciclaje debido a que la generación de material no orgánico que se puede reciclar, equivale aproximadamente a un 25,2 \% de los 4,1 millones de toneladas métricas, lo que significa que en el Ecuador anualmente se pueden reciclar aproximadamente 1 millón de toneladas métricas de desechos sólidos al año aproximadamente.

Adicionalmente y de acuerdo al Ministerio de Ambiente a través del Programa Nacional para la Gestión Integral de Desechos Sólidos se indica que el 24\% de Gobiernos Autónomos descentralizados Municipales (GADM ), ha emprendido procesos de separación en la fuente y recolección diferenciada, el 40\% de los GADM desarrollan procesos de aprovechamiento de residuos orgánicos y el $24 \%$ de los GADM desarrollan procesos de aprovechamiento de residuos inorgánicos, tales como papel, cartón, plástico, vidrio y chatarra. (Inicitiva regional para el reciclaje inclusivo, 2014-2015). En lo que respecta al servicio de recolección de residuos sólidos existe una cobertura nacional promedio del 84, 2\% para la zona urbana y del $54,1 \%$ en la zona rural, lo preocupante pero a la vez como un dato que puede ser visibilizado como una oportunidad para los NI de reciclaje es que el 35\% de los residuos sólidos de los GADM se los deposita en rellenos sanitarios bajo procesos técnicamente operados, no obstante el $65 \%$ se lo deposita sin un proceso técnico. (Inicitiva regional para el reciclaje inclusivo, 2014-2015)

\section{La empresa privada y su rol en el negocio inclusivo en la ciudad de Cuenca.}

En la ciudad de Cuenca este NI está concentrado en el Papel y Cartón debido a que no existen otras empresas que trabajen con material reciclado, la única Industria que tiene incorporado dentro de su cadena de abastecimiento la materia prima reciclada es la industria manufacturera de papel y cartón, sumándose a esto que según los datos antes presentados el papel y cartón representan al 9,4\% del total de residuos sólidos generados en un año a nivel nacional con una equivalencia de 0,39 millones de toneladas métricas al año, siendo de esta forma el segundo material reciclable luego del plástico.

Siendo la industria manufacturera de papel y cartón una empresa que se dedica a la realización de papel para la generación de empaques de cartón como proveedor externo 
e interno y a la vez pudiendo producir también empaques de cartón con el papel que la empresa producen, esto quiere decir la fabricación de cajas de cartón para diferentes tipos de productos como por ejemplo: línea blanca, licores, alimentos, flores, etc. Tanto para consumo nacional como para su exportación, si bien este análisis se centra en la ciudad de Cuenca esta industria es la única que cuenta con plantas en las ciudades de Guayaquil y Machala.

Esta industria constituyo en el valor agregado bruto para el año 2013 en miles de dólares la cantidad de 557.817,00 a nivel nacional y la provincia del Azuay aporta a esta cantidad con 51.443,72. (Ecuador, 2015), según el Ministerio del Ambiente en base al análisis para el periodo 2009-2013 indica que las exportaciones de residuos sólidos se concentran en chatarra, papel y cartón con un porcentaje de participación del 88,7 y 9,5 respectivamente. (Inicitiva regional para el reciclaje inclusivo, 2014-2015). La demanda a nivel del Ecuador de los años 2012 -2014 en lo que se refiere a cartón y papel ha crecido en un 300\% que equivale a un incremento de 60.870 toneladas en el año 2012 a 182.857 toneladas para el año 2014 esto según datos del (MAE-PNGIDS), en el año 2015, siendo el material reciclable con mayor crecimiento.

Esto sin duda refleja la importancia que tiene para la industria manufacturera de papel y cartón la inclusión de la población de la BPD por medio del NI de reciclaje de papel y cartón debido a la demanda de este recurso en el mercado nacional, a nivel del Ecuador las empresas que requieren papel y cartón como materia prima son: Papelera Nacional con su empresa Intercia, Cartopel, Grupo Surpapel, Incasa, las dos primeras tienen una participación de aproximadamente el $55 \%$ entre las dos, el grupo Surpapel e Incasa poseen alrededor del $22 \%$ y $15 \%$ respectivamente, adicionalmente según el Ministerio del Ambiente, la industria manufacturera de papel y cartón ha realizado inversiones considerables entre el periodo 2012-2014 que equivale a unos 150 millones de dólares. (Inicitiva regional para el reciclaje inclusivo, 2014-2015)

De acuerdo a proyecciones de la balanza comercial y la demanda de papel y cartón para el año 2014, el mercado nacional para esta industria necesita un aproximado de 28.571 toneladas mensuales lo que equivale a 342.852 toneladas al año, de este valor en promedio de $182.857(53,3 \%)$ toneladas anuales de papel y cartón son recuperadas en el Ecuador estando constituidas de $75 \%$ de cartón y el $25 \%$ de papel. Sin embargo para cubrir la demanda nacional se tiene que importar aproximadamente de 160.000 toneladas de papel y cartón, eso significa el $46,7 \%$ de la demanda anual de este mercado. (Inicitiva regional para el reciclaje inclusivo, 2014-2015)

Según (Inicitiva regional para el reciclaje inclusivo, 2014-2015) existe la viabilidad de recolectar a nivel nacional 5.000 toneladas adicionales por mes o lo que significa 60.000 
toneladas al año provocando un efecto inmediato en la disminución de las importaciones en un $37,5 \%$ y por ende en los costos de producción. En el Ecuador se recuperan 182.857 toneladas de papel y cartón por cada año, de las cuales aproximadamente 53.921 toneladas que equivalente al $(29,36 \%)$ son recuperadas por personas que se dedican al $\mathrm{NI}$ de reciclaje, de este valor el $5 \%$ le corresponde a la ciudad de Cuenca que significa unas $2.696,05$ toneladas.

Como se demuestra en los datos anteriores en el Ecuador así como también en la ciudad de Cuenca el $\mathrm{NI}$ de reciclaje de papel y cartón resulta ser muy atractivo para la industria manufacturera de papel y cartón por la demanda que existe a nivel nacional, la factibilidad de incrementar la recuperación de este material, las políticas a nivel nacional y de los GADM, lo cual se complementaría con el fortalecimiento de políticas empresariales que vean a esta actividad como una estrategia empresarial de creación de valor compartido (CVC), al cual se lo define como un elemento del concepto de los $\mathrm{NI}$ que intenta unir el mundo de los negocios con la sociedad, creando valor económico al mismo tiempo de crear valor para la sociedad. (Muñoz, 2013)

Los aspectos en los cuales se centra la CVC son: innovar el enfoque de productos y mercados, redefinir la productividad de la cadena de valor y generar el desarrollo de clústeres locales. El primero se refiere principalmente en identificar nuevas necesidades que tiene la sociedad en campos como salud, vivienda, medioambiente, etc. generando bienes y servicios innovadores que crean valor compartido, el segundo trata de mejorar el uso de los recursos, la energía, la logística, la cadena de distribución, la productividad de los empleados, reconfigurando las actividades de la cadena valor desde la perspectiva del valor compartido considerando dentro de esta como actores fundamentales a las personas de la BDP que pueden generar un $\mathrm{NI}$, el tercero trata de identificar deficiencias en áreas como logística, proveedores, canales de distribución, capacitación, instituciones educativas, etc. que contribuyan al desarrollo de nuevos clusters (o a la mejora de los existentes) en comunidades locales donde se encuentren ubicadas las empresas. (Muñoz, 2013)

La CVC cualquiera que sea el aspecto bajo el que se la aplique para que sea atractiva en una empresa se la debe entender como estrategia empresarial que busca un plan de acción que desarrolle ventaja competitiva y la acentúe de forma que ésta logre crecer y expandir su mercado reduciendo la competencia, articulando todas sus potencialidades de forma que la acción coordinada y complementaria de todos sus componentes contribuya al logro de objetivos definidos y alcanzables. (Vidal, 2011)

El pensar en la CVC para la industria manufacturera del papel y cartón propone una superación del concepto de la Responsabilidad Social Empresarial (RSE), ya que esta se 
"enfoca fundamentalmente en la reputación y solo tiene una conexión limitada del negocio" (Muñoz, 2013), mientras que la CVC no solo se centra en beneficios para la empresa e incluye a diferentes actores como son: el estado Ecuatoriano, la empresa privada y la sociedad, de donde se desprende según Adam Smith que no es suficiente el tener esclarecido el interés propio que es lo que buscan las empresas privadas al generar un negocio con el propósito de obtener ganancias para sus inversionistas, sino que aparte de esta motivación debe existir otras virtudes como la comprensión, la generosidad y el actuar en función del colectivo y bien de la sociedad. El estado también se encuentra inmerso debido a que en la óptica neoliberal se considera que las únicas que pueden afrontar los desafíos del mercado son las empresas, sin intervención del estado, debido a que lo creen no eficiente, por tal razón, el mismo considera que la única acción que puede realizar es desregular apartándose del camino de la empresa. (Crouch, 1996)

Este planteamiento implicaría que al aplicar la CVC en la industria manufacturera de papel y cartón su propósito consistiría en no solo obtener utilidades, sino también satisfacer las necesidades materiales y sociales de las personas de la BDP y de quienes se dediquen al NI de reciclaje de papel y cartón, esto significaría generar actividad empresarial no solo inspirados en la premisa de que el fin justifica los medios, es decir sin importar lo que tenga que hacer la empresa con el propósito de conseguir los resultados que desea, lo cual es muy peligroso porque de ser así la única razón de existir de una empresa será el obtener ganancias a costa del medio ambiente y de la sociedad.

No hay que olvidar que las organizaciones por si solas no tienen motivos y esto se debe a que las organizaciones están conformadas por personas quienes tienen sus propios intereses y motivaciones, que son las razones que harán actuar o no a una organización pensando en intereses propios o compartidos, esto se traduciría en la industria manufacturera del papel y cartón en un propósito compartido de no solo fabricar y proveer de los mejores productos que generen ingresos, sino también el producirlos considerando como proveedores y socios estratégicos a los recicladores y su organización la RENAREC en la ciudad de Cuenca, donde se evidenciaría no solo el interés de la empresa de obtener ganancias, sino también la prioridad que tendría por el bien social y medioambiental, esto implicaría para la empresa el pensar en los recicladores así como en el medio ambiente como una decisión de valor compartido, que se sustentaría en los principios morales de los inversionistas y accionistas de la empresa al considerar que sus principios como empresarios consisten en ayudar a la sociedad y medio ambiente porque sus principios así lo demandan.

Todo lo antes descrito permite llegar a la determinación que la generación del NI de reciclaje de papel y cartón en la población de la BDP con aplicación del concepto de valor 
compartido, invita a la construcción de un imperativo categórico, que según Kant es una regla moral universal que la misma sociedad se la impone a obedecerla por cuanto se la necesita como sociedad y consistiría en "la obtención de ingresos compartidos por parte de las empresas, sin perjuicio a la sociedad y el medio ambiente".

De esta manera la CVC servirá para fortalecer la partición activa del estado Ecuatoriano y la empresa privada, en concordancia con la perspectiva de (Sen, Capacidad y bienestar, 1996) en donde se define a la capacidad enfocada a las personas en la habilidad real para lograr funcionamientos valiosos para su vida, como: el estar nutrido, tener acceso a la educación o buena salud, etc. Pero para conseguirlo dependerá de la ayuda del estado y de la empresa privada debido a que estos proporcionaran el entorno que permita la consecuciones de estos funcionamientos al brindar las capacidades necesarias en la población de la BDP que se dedican al NI de reciclaje, proporcionando un enfoque para el igualitarismo en presencia de privaciones elementales, esto quiere decir que no toda la población tendrá acceso ciertos productos o servicios que en algunos de los casos se podrían considerar como no prioritarios, pero si a cubrir sus capacidades básicas, es decir mejorar el estado social de las personas y las cosas que logra hacer o ser al vivir.

\section{Conclusiones}

La información presentada sobre la BDP y el mercado potencial en diferentes categorías que se genera en función a ella en América Latina y el Caribe y a nivel del Ecuador permite considerar la aplicación y realización de una profundización a futuro mediante una investigación científica del concepto de NI inclusivo en la Ciudad de Cuenca, que brindaran no solo a la Industria Manufacturera del Papel y Cartón sino también al resto de industrias las posibilidades de generación de estrategias empresariales que permitan obtener a la par ingresos económicos y una contribución a la sociedad, en cooperación directa con el estado Ecuatoriano que tiene un rol preponderante en el fortalecimiento y éxito del $\mathrm{NI}$, al regular la empresa privada mediante la generación de políticas públicas incluyentes, sin desmedro de la eficiencia y eficacia necesarias en el ámbito de los negocios.

Aunque este claro el potencial de los $\mathrm{NI}$ en el Ecuador y en la ciudad de Cuenca, todavía queda mucho camino por recorrer en el país, en materia de superar paradigmas por parte de empresarios, estado y la sociedad, quienes estigmatizan a la población de la BDP al considerarla incapaz de ser vista como: socios empresariales, proveedores o como consumidores, debido al hecho de no disponer los suficientes recursos económicos para ser considerados parte de la actividad empresarial, esto implica un gran reto en el Ecuador, 
debido a que existen las condiciones idóneas para poder aplicar el concepto de NI en la $\mathrm{BDP}$, no obstante esto dependerá de una nueva visión por parte de todos los actores que intervienen en los $\mathrm{NI}$.

La aplicación de los NI como una estrategia empresarial implica no solo mirar el bienestar de la empresa, sino también considerar a la par el beneficio para la sociedad, donde es necesario una visión de valor compartido, evitando idealizar a esta estrategia como una única solución para todos los problemas económicos y sociales, sin desmerecer las oportunidades que brinda a las empresas para actualizar la perspectiva a través de la cual conciben a la estrategia, en la que solo se pensaba en los ingresos, mientras que el bienestar de la sociedad era abordado desde una perspectiva con fines únicamente de publicidad, antes que de una verdadera intervención de impacto con resultados medibles a corto, mediano o largo plazo.

De su parte los recicladores por medio de su organización han encontrado la manera de hacer visible su actividad y la contribución a la cadena de valor de la industria manufacturera de papel y cartón al convertirse en proveedores de materia prima accediendo de esta manera a la reducción de costos de producción, el organizarse ha permitido la consecución de algunos logros en materia de convenios, ordenanzas y políticas que van en beneficio de su organización y que permiten seguir fortaleciendo su actividad, a esto se suma el potencial identificado en lo que respecta al reciclaje no solo en la ciudad de Cuenca sino en todo el Ecuador. 


\section{REFERENCIAS}

AVINA. (2010). Negocios y Mercados Inclusivos. Definición y Marco Conceptual para el trabajo de AVINA. Montevideo.

Banco Interamericano de Desarrollo. (2015). Un mercado creciente de Usd. \$750 mil millones, Descubriendo oportunidades en la base de pirámide en América Latina y el Caribe. Banco Interamericano de Desarrollo.

Brown, M. (1992). La ética en la empresa. Estrategia para la toma de decisiones. Buenos Aires: Paidós.

Crouch, C. (1996). El problema de la empresa. En La democracia post-liberal (págs. 331-349). Madrid: Tezanos, Felix.

D’Odorico, M. G. (2015). Material de Seminario sobre cuestiones ético profecionales del científico. Rosario- Argentina.

Diaz Cáceres, N., \& Castaño Carlos, A. (2013). El Valor Compartido Como Nueva Estrategia de Desarrollo Empresarial. Internacional Journal of Good Conscience, 82-100.

Ecuador, B. C. (20 de 9 de 2015). http://www.bce.fin.ec/index.php/component/k2/item/293cuentas-provinciales. Recuperado el 20 de septiembre de 2015, de http://www.bce.fin.ec/ index.php/component/k2/item/293-cuentas-provinciales: http:/www.bce.fin.ec/index.php/ component/k2/item/293-cuentas-provinciales

INEC. (1 de septiembre de 2015). http://www.ecuadorencifras.gob.ec/. Recuperado el 1 de septiembre de 2015, de http://www.ecuadorencifras.gob.ec/: http:/www.ecuadorencifras. gob.ec/

Inicitiva regional para el reciclaje inclusivo. (2014-2015). Reciclaje Inclusivo y recicladores de base en el Ecuador. Don Bosco.

Licandro, O. (2013). El rol de las alianzas intersectoriales en la creacion de negocios inclusivos con la base de la pirámide, un análisis de la experiencia uruguaya mediante el estudio de casos.

Licandro, O., \& Pardo, L. (2013). Experiencias de Negocios Inclusivos en el Uruguay. Montevideo: Universidad Católica del Uruguay.

Muñoz, J. (2013). Ética empresarial, Responsabilidad Social Corporativa (RSC) y Creación de Valor Compartido (CVC). Globalización, Competitividad y Gobernabilidad, 76-88.

Netherlands Development Organisation (SNV); World Business Council for Sustainable Development. (2010). Negocios Inclusivos: Creando valor en América Latina.

Pineda Escobar, M., \& Falla Villa, P. (2016). Los Negocios Inclusivos como fuente de trabajo de calidad para pequeñas empresarias en condición de pobreza: un estudio exploratorio en el municipio de Apartadó, Colombia. Equidad \& Desarrollo, 179-208. 
Prahalad, C. K., \& Hammond, A. (2005). Atender a los pobres del mundo, rentablemente. Harvard Business Review, 87-99.

Prahalad, C., \& L.Hart, S. (2002). The Fortune at the Bottom of the Pyramid. Booz Allen Hamilton.

Renarec. (16 de septiembre de 2015). https://renarec.wordpress.com/. Recuperado el 20 de septiembre de 2015, de https://renarec.wordpress.com/: https://renarec.wordpress.com/

Sen, A. (1996). Capacidad y bienestar. Mexico.

Sen, A. (2003). Qué impacto puede tener la ética. TRABAJO PRESENTADO EN EL SEMINARIO INTERNACIONAL “ETICA T DESARROLLO”. BANCO INTERAMERICANO DE DESARROLLO.

Vidal, I. (2011). El principio de valor compartido de Porter y Kramer. Boletin del Centro de Investigación y Economía, 1-5.

Ynoub, R. (2015). Cuestión de Método: Aportes para una metodología crítica. México: Cengage Learning Editores, S.A. 